\title{
Electron microscopic study of intercellular junctions in human gastric mucosa with special reference to their relationship to gastric ulcer
}

T Ohkusa, M Yamamoto, K Kataoka, T Kyoi, F Ueda, H Fujimoto, M Sasabe, Y Tamura, H Hosoi, S Tokoi, N Sasaki, Y Saito

\begin{abstract}
The development of gap junctions in the human gastric mucosa has been examined to see if there is any relation to gastric ulcer. Freeze fracture replicas were prepared from the endoscopic biopsy specimens of 20 patients with gastric ulcer (15 men and five women, aged 49 (13) years) and seven healthy volunteers (four men and three women, aged 41 (19) years). Large fractured areas of lateral cell membranes of surface mucous cells were observed randomly at a direct magnification of 15000 using electron microscopy. Small gap junctions were observed between gastric surface mucous cells in all healthy volunteers. Gap junctions in the patients with gastric ulcer were significantly fewer than in the healthy volunteers. In addition, gap junctions in patients with recurrent ulcer were significantly fewer than in those with first onset ulcer. There was no obvious relationship between age and the development of gap junctions in patients with gastric ulcer or in healthy volunteers. In areas of intestinal metaplasia, gap junctions were occasionally seen between absorptive cells of the villi, but not in the lateral membranes of goblet cells. These findings suggest that loss of intercellular communication via gap junctions is associated with gastric ulcer formation.

(Gut 1993; 34: 86-89)
\end{abstract}

Most cells continue their vital activities, such as differentiation and proliferation, while maintaining homeostasis through intercellular communication via gap junctions. ${ }^{1}$ Gap junctions are distributed in a wide variety of tissues, with the possible exceptions of adult skeletal muscles and most neurones. ${ }^{2}$ Gap junctions in surface mucous cells of the normal gastric mucosa have been demonstrated in animals, including rats and mice, ${ }^{3-6}$ but their relation to gastric mucosal injury has not been reported. It has been shown that experimental anoxia and a metabolic uncoupler, 2,4-dinitrophenol, cause structural changes in gap junctions, characterised by a tighter hexagonal aggregation of the gap junctional particles in the stomach and other tissues. $^{5-8}$ Therefore, we decided to compare gap junctions between gastric surface mucous cells of gastric ulcer patients with those of healthy volunteers.

In the present study, the presence of gap junctions in surface mucous cells in endoscopic biopsy specimens from patients with gastric ulcer was investigated by freeze fracture methods. The roles of gap junctions in the formation of gastric ulcers and the maintenance of tissue homeostasis are discussed.

\section{Methods}

PATIENTS AND HEALTHY VOLUNTEERS

The subjects were 20 Japanese patients with gastric ulcers (15 men and five women, aged 49 (13) years) who visited our hospital for gastroendoscopic examination. Gastric ulcers caused by steroids and non-steroidal anti inflammatory drugs were excluded. These patients received no medication during the month before gastroendoscopy. As shown in Table I, the ulcer was in an active stage in 11 patients, healing stage in eight patients, and scar stage in one patient. The endoscopic staging of ulcers was done according to the standard Japanese protocol: ${ }^{9}$ Briefly, the active stage of ulcer has a crater without surrounding regenerative mucosa, the healing stage of ulcer has an area of deformity, a patch of erythema, and a small residual crater, and the scar stage of ulcer has radiating folds but no crater. Twelve patients (nine men and three women, aged 52 (10) years) had recurrent ulcers, and eight patients (six men and two women, aged 45 (15) years) had first onset ulcers. The diagnosis of recurrent ulcers was made from the past history and gastrofibrescopic examination.

The study was approved by the ethics committee of our university and informed consent was obtained from all patients and healthy volunteers.

\section{FREEZE FRACTURE ANALYSIS}

Tissue specimens were taken with biopsy forceps (FB-25K, Olympus, Tokyo, Japan) from the border of the gastric ulcer, and 5-10 cm away from the ulcer in the endoscopically normal gastric mucosa. Grossly normal appearing gastric biopsy specimens were taken from healthy volunteers.

The specimens were fixed with $3 \cdot 7 \%$ formal-

TABLE I Characteristics of patients with gastric ulcer and healthy volunteers

\begin{tabular}{lclcl}
\hline $\begin{array}{l}\text { Patients } \\
\text { stage of ulcer }\end{array}$ & Male & Female & Total & $\begin{array}{l}\text { Average } \\
\text { age }\end{array}$ \\
\hline Active & 10 & 1 & 11 & $51(12)$ \\
Healing & 4 & 3 & 7 & $44(12)$ \\
Scar & 1 & 1 & 2 & $61(11)$ \\
Total & 15 & 5 & 20 & $49(13)$ \\
Healthy volunteers & 4 & 3 & 7 & $41(19)$ \\
\hline
\end{tabular}

$\star$ Mean (SD).
Correspondence to:

Toshifumi Ohkusa, MD, Firs Medicine, of Internal Tokyo Medical and Dental University, Yushima 1-5, Japan.

Accepted for publication 3 July 1992 
Figure 1: Lateral plasmalemmas of gastric surface mucous cells from a healthy volunteer. A small gap junction is seen as an aggregation of

intramembranous particles (arrow). L: gastric lumen. Bar represents 0.5 $\mu \mathrm{m}$.

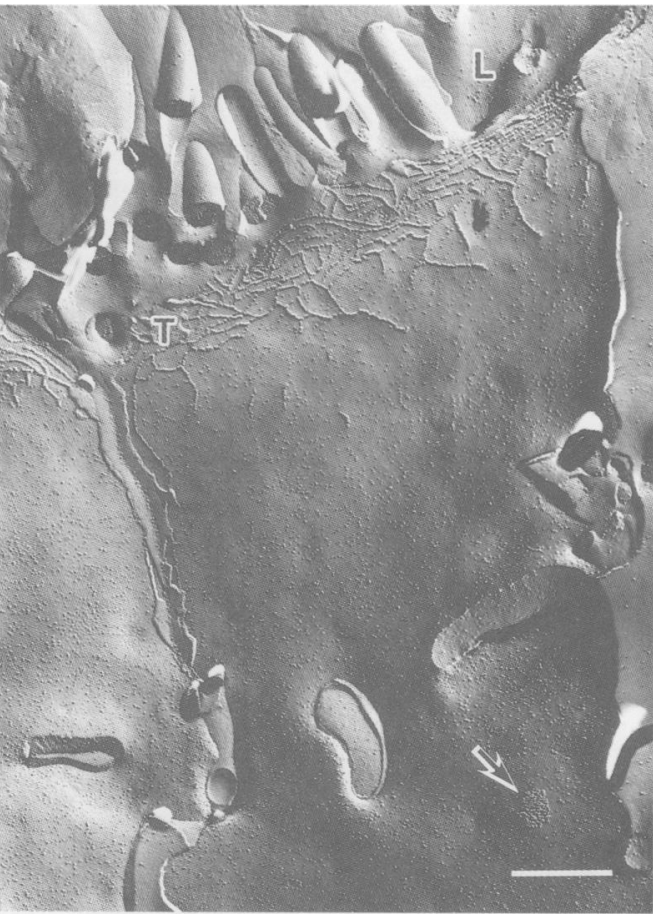

dehyde and $1 \%$ glutaraldehyde in $0 \cdot 1 \mathrm{M}$ cacodylate buffer (pH 7•4). After immersion in $40 \%$ glycerin solution at room temperature, they were placed on a copper stage and frozen with liquid nitrogen. The specimens were fractured at $-110^{\circ} \mathrm{C}$ in a JEOL JFD 7000 freeze fracture apparatus and shadowed with platinum carbon without etching, then digested in filtered commercial bleach. The replicas were washed twice in distilled water, mounted on grids and examined in a Hitachi H-500 electron microscope. All morphologic measurements were done without knowledge of the tissue source. The large fractured area containing the lateral cell membranes of surface mucous cells was observed and photographed randomly at a direct magnification of $\times 15000$. The number of gap junctions was scored as follows: grade - , no gap junctions in gastric surface mucous cells in over 50 electron micrographs; grade + , more than one gap junction in 11-50 micrographs; grade $2+$, more than one gap junction in 1-10 micrographs. The criterion for a gap junction was a minimum of 20 membrane particles in a plaque.

\section{STATISTICAL ANALYSIS}

Gastroscopy reports and histopathology were coded independently in different departments.

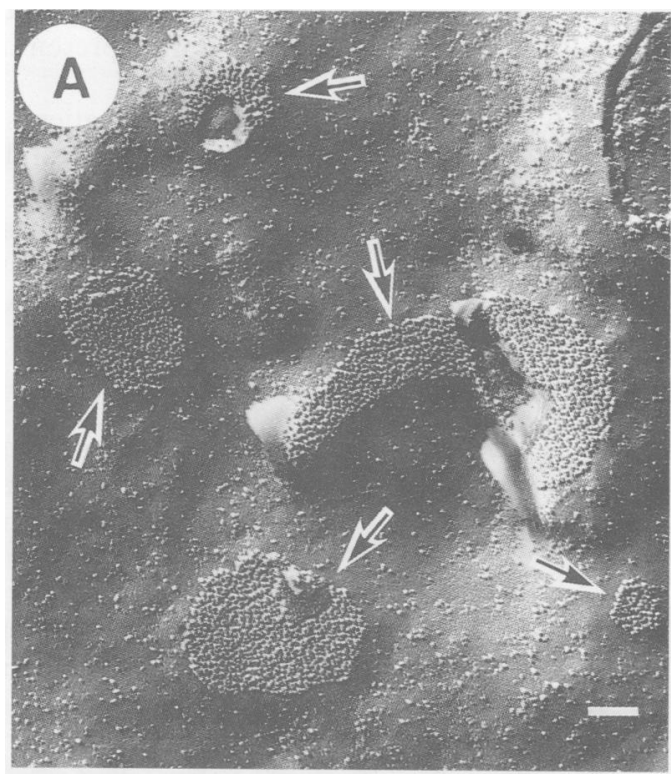

The results were analysed by the $\chi^{2}$ test. A probability value of $<0.05$ was considered to be statistically significant.

\section{Results}

GAP JUNCTION

In healthy volunteers, small gap junctions were often identified on the lateral plasmalemma of gastric surface mucous cells, mainly at the level of the cell nucleus (Figs 1, 2). On the P-face, a gap junction was recognised as an aggregation of intramembranous particles, 8 to $9 \mathrm{~nm}$ in diameter (Fig 2B). Complementary pits were seen on the E-face.

The frequency of gap junctions in the gastric surface mucous cells of the patients with gastric ulcers and of healthy volunteers is shown in Table II. The patients with gastric ulcer had significantly fewer gap junctions than did the healthy volunteers. The frequency of gap junctions at the edge of the gastric ulcer was almost the same as that in the endoscopically normal mucosa of the gastric fundus or antrum at a distance from the ulcer.

The patients with recurrent ulcers had significantly fewer gap junctions in the ulcer

TABLE II Gap junction frequency of surface mucous cells in patients with gastric ulcer and healthy volunteers

\begin{tabular}{llll}
\hline Gap junction frequency ${ }^{\star} S$ & - & + & $2+$ \\
\hline Gastric Ulcer $\dagger$ & 7 & 9 & 4 \\
Healthy volunteers $\ddagger$ & 0 & 1 & 6 \\
\hline
\end{tabular}

${ }^{\star}$ Gap junction frequency $(\times 15000)$

- , no junction in $>50$ views

,$+>1$ junction in $11-50$ views
$2+,>1$ junction in $1-10$ views.

tAge 49 (13). Gastric surface mucous cells from edge of ulcer. $\neq$ Age 41 (19). Gastric surface mucous cells of endoscopically

$\S \dagger$ and $\neq$ significant difference by $\chi^{*}$ test $(p<0.05)$. (arrows). (B) Higher junction showing $a$ disordered array of 8-9 $\mathrm{nm}$ central pit on the gap junctional particles. Bar represents $0 \cdot 1 \mu \mathrm{m}$.
At the level of gap junctions are seen

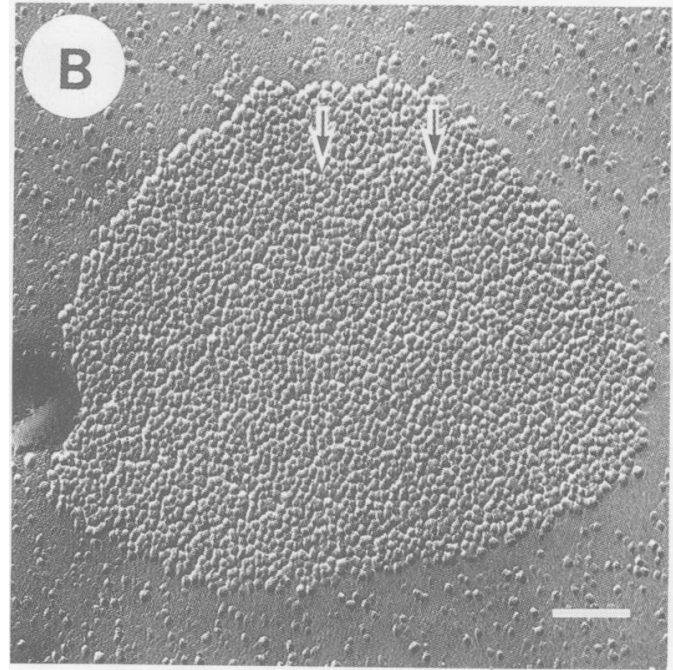


TABLE III Gap junction frequency of surface mucous cells in patients with recurrent ulcer and first onset ulcer

\begin{tabular}{llll} 
Gap junction frequency $* \mathbb{S}$ & - & + & $2+$ \\
\hline Recurrencet & 7 & 5 & 0 \\
First onset $\neq$ & 0 & 4 & 4 \\
\hline
\end{tabular}

${ }^{\star}$ Gap junction frequency $(\times 15000)$

- , no junction in $>50$ views

,$+>1$ junction in $11-50$ views

$2+,>1$ junction in $1-10$ views.

†Age 52 (10)

$\ddagger$ Age $45(15)$.

$\S+$ and $\ddagger$ significant difference by $\chi^{2}$ test $(\mathrm{p}<0 \cdot 05)$.

TABLE IV Gapjunction frequency of surface mucous cells in patients with gastric ulcer; variations by stage of ulcer

\begin{tabular}{|c|c|c|c|}
\hline Gap junction frequency ${ }^{\star}$ & - & + & $2+$ \\
\hline Activet & 4 & $\begin{array}{l}5 \\
4\end{array}$ & $\begin{array}{l}2 \\
2\end{array}$ \\
\hline $\begin{array}{l}\text { Healing } \\
\text { Scar } \$\end{array}$ & $\begin{array}{l}2 \\
1\end{array}$ & $\begin{array}{l}4 \\
0\end{array}$ & $\begin{array}{l}2 \\
0\end{array}$ \\
\hline
\end{tabular}

${ }^{\star}$ Gap junction frequency $(\times 15000)$

- , no junction in $>50$ views

,$+>1$ junction in $11-50$ views

†Age 51 (12)

$\ddagger$ Age $44(12$

SAge $61(11)$

margin than did the patients with first onset ulcer (Table III).

There was no difference in the frequency of gap junctions among the three stages of ulcer active, healing, and scar (Table IV).

As shown in Figure 3, the frequency of gap junctions in both patients with gastric ulcer and healthy volunteers appeared to be independent of age.

Intestinal metaplasia was seen in the biopsy specimens from eight patients with gastric ulcer. The frequency of gap junctions between the absorptive cells in the area of the intestinal metaplasia was low (Fig 4), and gap junctions were not observed in the lateral membranes of goblet cells.

\section{TIGHT JUNCTIONS}

In the normal gastric mucosa of healthy volunteers, a tight junction between surface mucous

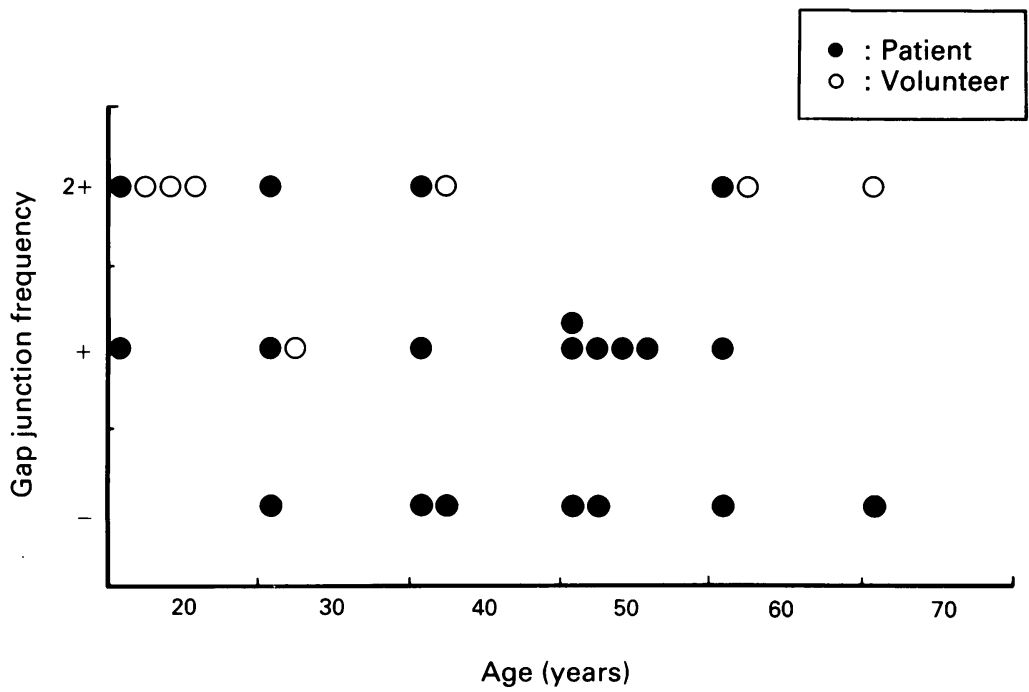

Figure 3: Frequency of gap junctions in patients with gastric ulcer and healthy volunteers $(n=27)$. (1): Gap junction frequency; $-:$ no junction in $>50$ views; $+:>1$ junction in 11-50 views; $2+:>1$ junctions in $1-10$ views. The age ranges plotted are: $20-29,30-39,40-49$, $50-59,60-69$, and $>70$ years.

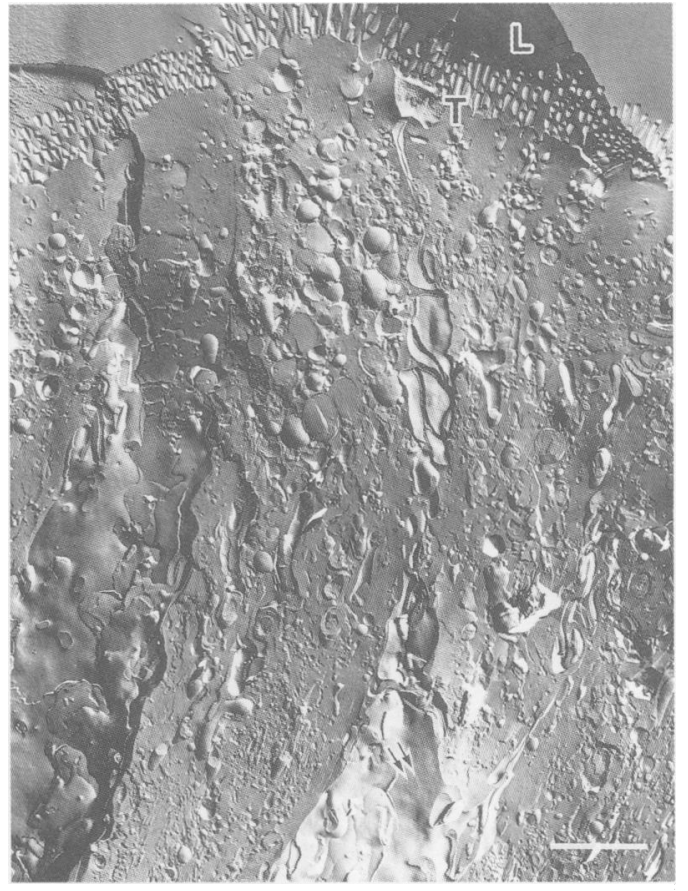

Figure 4: Intestinal metaplasia in a patient with gastric ulcer. $A$ small gap junction is seen in the lateral plasmalemma of an absorptive cell (double arrow). L: gastric lumen. T: tight junction. Bar represents $2 \mu \mathrm{m}$.

cells was composed of a network of 5-7 junctional strands presenting a honeycomb appearance (Fig 5A).

In 10 of 13 patients with gastric ulcer, tight junctions often exhibited discontinuity and decreased numbers of tight junctional strands, and extensions of apical tight junctional strands to the base of a cell were occasionally seen (Figs $5 B, C)$.

\section{Discussion}

Most cells carry on their vital activities, such as differentiation and proliferation, while maintaining homeostasis through intercellular communication via gap junctions.' The results of our freeze fracture method clearly showed significantly fewer gap junctions between gastric surface mucous cells in patients with gastric ulcer than in

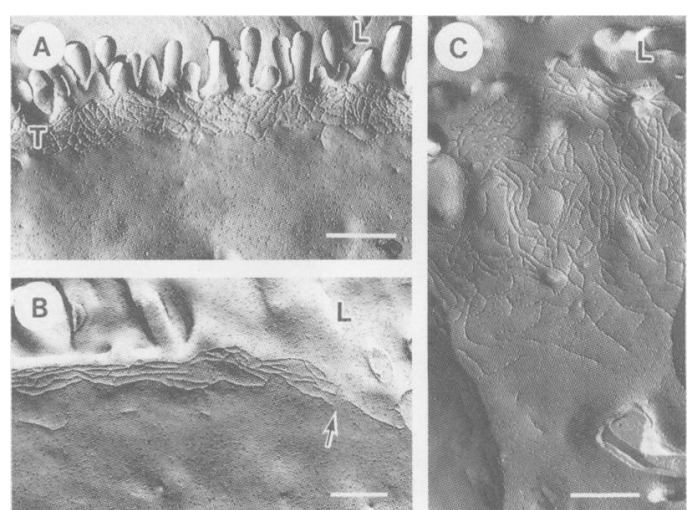

Figure 5: Lateral plasmalemmas of gastric surface mucous cells in a healthy volunteer $(A)$ and a patient with gastric ulcer $(B, C)$. (A) tight junctions $(T)$ consist of networks of five to seven junctional strands. (B) Discontinuity (arrow) and decrease in number of tight junctional strands are seen. $(C)$ Hyperplastic tight junctions (apical tight junctional strands extending over the lateral plasma membrane) are seen. $L$ : gastric lumen. Bar represents $0.5 \mathrm{um}$. 
healthy volunteers. There was no association between gap junction frequency and age. These morphological findings suggest that the intercellular communication mediated by gap junctions involved in the local homeostasis of the gastric mucosa is decreased in patients with gastric ulcer and that this decrease may be closely related to gastric ulcer formation. There was no association, however, between gap junction frequency and endoscopically evaluated ulcer stage. There was also no difference in gap junction frequency between biopsy specimens from the ulcer margin and those from grossly normal regions at a distance from the ulcer. Interestingly, gap junctions in patients with recurrent ulcer were much fewer than in patients with first onset ulcer. These results suggest that the loss of intercellular communication mediated by gap junctions may be associated with the recurrence of gastric ulcers. Therefore, a reduction in the number of gap junctions may be one of the pathogenic factors in chronic or recurrent gastric ulcers.

Gap junctions were occasionally found between absorptive cells in the villi, but not on the lateral cell membranes of goblet cells, in intestinal metaplasia of the gastric mucosa. The findings in the present study are in agreement with those in the epithelium of the rat small intestine described by Kataoka et al. ${ }^{10}$

The normal gastric mucosa can prevent bypass diffusion of potentially noxious substances from the gastric lumen. Morphologically, this barrier is provided principally with tight junctions between gastric surface mucous cells. ${ }^{11} \mathrm{Claude}^{12}$ has suggested that there is an exponential relationship between the number of tight junctional strands and transepithelial resistance. Meyer et $a l^{13}$ reported that tight junctions in the canine gastric mucosa were significantly damaged by exposure to aspirin. It has been thought that damage of the tight junctions is morphologically represented by focal discontinuities and veriability in the numbers of tight junctional strands. In our study, tight junctions were often characterised by discontinuity and decreased numbers of tight junctional strands.
In addition, hyperplastic tight junctions and free ending basal extensions of tight junctional strands were occasionally seen. These findings are in agreement with those of Posalaky et al, ${ }^{1+}$ who observed discontinuity and hyperplasia in tight junctions in gastritis and gastric ulcer biopsies.

In conclusion, using the freeze fracture method, we have shown significantly fewer gap junctions between gastric surface mucous cells in ulcer patients than in healthy volunteers. These findings suggest that the loss or decrease of gap junctions in gastric surface epithelium may be associated with gastric ulcer formation via changes in intercellular communication capacity.

This work was presented in part at the 1991 Annual Meeting of the American Gastroenterological Association and has been published in abstract form (Gastroenterology 1991; 100: A135).

1 Loewenstein WR. Junctional intercellular communication: the cell to cell membrane channel. Physiol Rev 1981; 61: 829-913.

2 Larsen WJ. Biological implications of gap junction structure, distribution and composition: A review. Tissue Cell 1983; 15: 645-71.

3 Shibata Y, Izumi T, Yamamoto T. Tissue-specific granularity of gap junction cytoplasmic surfaces revealed by rapid-
freeze, deep-etch replicas. Anat Rec 1989; 223: 113-20.

4 Kataoka K, Maesako J, Yamamoto M, Toyota T. Intercellular junctions in the gastric mucosa of mice and rats. Proceedings junctions in the gastric mucosa of mice and rats. Proceedings 2877 .

5 Peracchia C. Gap junctions Structural changes after uncoupling procedures. $\mathcal{F}$ Cell Biol 1977; 72: 628-41

6 Peracchia C. Structural correlates of gap junction permeation. Int Rev Cytol 1980; 66: 81-146.

7 Hoyt RH, Cohen ML, Corr PB, Saffitz JE. Alterations of intercellular junctions induced by hypoxia in canine myocardium. Am F Physiol 1990; 258: H1439-48.

8 De Maziere AMGL, Scheuermann DW. Structural changes in cardiac gap junctions after hypoxia and reoxygenation: a
quantitative freeze-fracture analysis. Cell Tissue Res 1990; quantitative

9 Sakita T, Miwa T. Endoscopic findings of gastric ulcer (in Japanese). $\mathcal{F} p n \mathcal{F}$ Gastroenterol 1970; 67: 984-9.

10 Kataoka K, Tabata J, Yamamoto M, Toyota T. The association of gap junctions with large particles in the crypt epithelium of the rat small intestine. Arch Histol Cytol 1989, 52: 81-6.

11 Powell DW. Barrier function of epithelia. Am F Physiol 1981; 241: G275-88.

12 Claude P. Morphological factors in influencing transepithelial permeability: a model for the resistance of the zonula occludens. F Membr Biol 1978; 39: 219-32.

13 Meyer RA, Mcginley D, Posalaky Z. Effects of aspirin on tight junction structure of the canine gastric mucosa. Gastroenterology 1986; 91: 351-9.

14 Posalaky Z, Posalaky I, McGinley D, Meyer RA. The gastric mucosal barrier: tight junction structure in gastritis and ulcer biopsies. Virchows Arch 1989; 414: 217-2. 